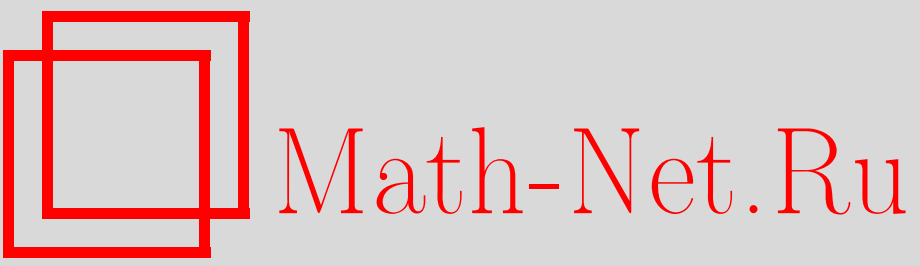

М. Л. Гервер, Несколько простых теорем о расположении корней, УМН, 1996, том 51, выпуск 3, 191-192

DOI: https://doi.org/10.4213/rm979

Использование Общероссийского математического портала Math-Net.Ru подразумевает, что вы прочитали и согласны с пользовательским соглашением

http://www.mathnet.ru/rus/agreement

Параметры загрузки:

IP : 3.82 .47 .9

26 апреля 2023 г., 14:46:27 


\title{
НЕСКОЛЬКО ПРОСТЫХ ТЕОРЕМ О РАСПОЛОЖЕНИИ КОРНЕЙ
}

\author{
М. Л. ГЕРВЕР
}

Рассмотрим множество $K_{n}$ тригонометрических многочленов

$$
\begin{gathered}
Q(x)=c_{0}+c_{1} \cos x+c_{2} \cos 2 x+\cdots+c_{n} \cos n x, \\
0<c_{0}<c_{1}<c_{2}<\cdots<c_{n} .
\end{gathered}
$$

Согласно $[1$, часть 1 , отдел 3 , задача 185, с. 149,342$]$ любой тригонометрический многочлен из $K_{n}$ имеет $n$ вещественных простых корней в интервале $0<x<\pi$ (поскольку полином

$$
P(z)=c_{0}+c_{1} z+c_{2} z^{2}+\cdots+c_{n} z^{n}
$$

с коэффициентами вида (2) имеет в единичном круге $n$ нулей).

В заметке формулируются теоремы, уточняющие этот результат. Первые две из них дают ответ, например, на такой

Вопрос. Возьмем любъе два тригонометрических многочлена $Q_{1}$ и $Q_{2}$ из множества $K_{n}$ и отметим корни одного красными точками, а корни другого - синими. Обозначим через $k(n)$ максимальное (по всем парам $\left.Q_{1}, Q_{2}\right)$ число красных точек, идущих подряд (не разделенных синими). Чему равно $k(n)$ ?

Базис $f_{j, n+1}$. Положим $r_{1}=c_{n}-c_{n-1}, \ldots, r_{n}=c_{1}-c_{0}, r_{n+1}=c_{0}$,

$$
f_{j, n+1}(x)=\sum_{s=n+1-j}^{n} \cos s x=\frac{\sin j x / 2 \cos (2 n+1-j) x / 2}{\sin x / 2}, \quad 1 \leqslant j \leqslant n+1,
$$

и запишем (1) в виде линейной комбинации функций (4) с положительными (ввиду (2)) коэффициентами: $r_{1} f_{1, n+1}(x)+r_{2} f_{2, n+1}(x)+\cdots+r_{n} f_{n, n+1}(x)+r_{n+1} f_{n+1, n+1}(x)$.

Матрица $\mathscr{M}_{n}$. Составим матрицу $\mathscr{M}_{n}$ из корней функций (4) на отрезке $[0, \pi]$. Сумма кратностей этих корней равна $n$ при каждом $j(1 \leqslant j \leqslant n+1)$, и все корни - не более, чем двукратные. Упорядочим корни $f_{j, n+1}(x)$ на $[0, \pi]$ по возрастанию (повторяя двукратные корни дважды) и поместим в $j$-ю строку матрицы $\mathscr{M}_{n}$.

Лемма. При всех $n$ и $k(1 \leqslant k \leqslant n-1)$ любой әлемент а из $(k+1)$-го столбца матриць $\mathcal{M}_{n}$ и любой әлемент $b$ из ее $k$-го столбца связань неравенством $a \geqslant b$.

Интервалы $\left(a_{k, n}, b_{k, n}\right)$. Произвольно фиксируем $n$ и при каждом $k(1 \leqslant k \leqslant n)$ выберем в $k$-м столбце матрицы $\mathscr{M}_{n}$ минимальныцй и максимальный әлементы $a_{k, n} u b_{k, n}$. По лемме интервалы $\left(a_{k, n}, b_{k, n}\right), 1 \leqslant k \leqslant n$, попарно не пересекаются и разделены отрезками или точками $b_{k, n} \leqslant x \leqslant a_{k+1, n}, 1 \leqslant k \leqslant n-1$. Положим $b_{0, n}=0, a_{n+1, n}=\pi$.

Tеорема 1. Все $Q(x) \in K_{n}$ имеют ровно по одному корню в каждом из интервалов $\left(a_{k, n}, b_{k, n}\right), 1 \leqslant k \leqslant n$. При $b_{k, n} \leqslant x \leqslant a_{k+1, n}(1 \leqslant k \leqslant n)$ все $Q(x) \in K_{n}$ положительнь, если $k$ четно, и отрицательнь, если $k$ нечетно.

СлЕДСтвиЕ. Ответ на поставленный выше вопрос: $k(n) \leqslant 2$.

Из теоремы 2 следует: $k(1)=1, k(n)=2$ при всех $n>1$.

ЗАмечАниЕ. Если требовать лишь, чтобы у рассматриваемых тригонометрических многочленов все корни были вещественными, то $k(n) \rightarrow \infty$ при $n \rightarrow \infty$.

Область $G_{n}$. Корни $x_{k} \in\left(a_{k, n}, b_{k, n}\right)$ тригонометрического многочлена $Q \in K_{n}$ удобно изображать в виде точки $X=X(Q)=\left\{x_{k}\right\} \in \Pi_{n}$, где $\Pi_{n}$ - прямое произведение интервалов $\left(a_{k, n}, b_{k, n}\right), 1 \leqslant k \leqslant n$. Поскольку $X(Q) \equiv X(\lambda Q)$ при $\lambda \neq 0$, нормируем тригонометрические многочлены и будем считать, что в (1)

$$
0<c_{0}<c_{1}<c_{2}<\cdots<c_{n}=1 .
$$

Работа выполнена при финансовой поддержке Российского фонда фундаментальных исследований (грант № 94-01-01350). 
Теорема 2. Точки $X(Q), Q \in K_{n}$, образуют область $G_{n} \in \Pi_{n}$, гомеоморфную симплексу (5). Ее граница $\partial G_{n}$ гомеоморфна граниче симплекса (5) и состоит из точек $X(f)$, әде $f=\sum_{j=1}^{n+1} r_{j} f_{j, n+1}(x), r_{j} \geqslant 0, \prod_{j=1}^{n+1} r_{j}=0, \sum_{j=1}^{n+1} r_{j}>0$.

Аналогичные теоремы верны для множеств $K_{0, n}$ и $K_{1, n}$ тригонометрических многочленов $c_{0}+c_{1} \cos 2 x+\cdots+c_{n} \cos 2 n x$ и $c_{0} \cos x+c_{1} \cos 3 x+\cdots+c_{n} \cos (2 n+1) x$ с коэфффициентами (2), для таких же множеств тригонометрических многочленов по синусам с положительными возрастающими коэффициентами и некоторых других множеств.

Общая ситуация описывается следующей теоремой. Рассмотрим множество $V$ непрерывных функций на отрезке $[0, L]$, обладающее двумя свойствами:

А. $V$ выпукло, т.е. функция $p f+q g \in V$ для любых $f, g \in V$ и любых $p, q>0, p+q=1$.

В. Каждая функция $f \in V$ имеет $n$ корней в интервале $(0, L)$, где $n$ - одно и то же для всех $f$ из $V$; эти корни - простые, и вблизи нуля $f(x)>0$ при $x>0$.

ПримеРы. Множества $K_{n}, K_{0, n}$ и $K_{1, n}$, очевидно, выпуклы. Свойство В для $K_{0, n}$ и $K_{1, n}$ доказывается заменой полинома $(3)$ на $P\left(z^{2}\right)$ и на $z P\left(z^{2}\right)$.

Теорема 3. Существуют такие отрезки (или точки)

$$
\left[a_{k}, b_{k}\right], \quad 1 \leqslant k \leqslant n, \quad 0 \leqslant a_{1} \leqslant b_{1} \leqslant \cdots \leqslant a_{n} \leqslant b_{n} \leqslant L,
$$

что любая функция $f \in V$ имеет в них ровно по одному корню.

ДоказАТЕЛЬСтво. Возьмем любые $f$ и $g$ из $V$ и обозначим через $x_{k}$ и $y_{k}, 1 \leqslant k \leqslant n$, их корни в интервале $(0, L): 0<x_{1}<x_{2}<\cdots<x_{n}<L ; 0<y_{1}<y_{2}<\cdots<y_{n}<L$. Тогда $x_{j} \leqslant y_{j+1}$ и $y_{j} \leqslant x_{j+1}$ при всех $j$ от 1 до $n-1$ (иначе была бы невозможна гомотопия $(1-t) f+t g$, переводящая все $x_{k}$ в $\left.y_{k}\right)$, и остается положить $a_{k}=\inf x_{k}, b_{k}=\sup x_{k}$ (по всем $f \in V)$.

ЗАмечАниЕ. Приведенное доказательство (с незначительными изменениями) годится для леммы к теореме 1.

Специфика $K_{n}, K_{0, n}, K_{1, n}$ и т. п. (по сравнению с множествами $V$ общего вида) - существование базиса типа (4). Благодаря его наличию теорема 1 и ее модификкации приводят, несмотря на свою крайнюю простоту, к довольно точным асимптотическим оценкам (стандартные доказательства которых требуют вычислений). В качестве примера рассмотрим полиномы Лежандра

$$
L_{0}(x)=1, \quad L_{1}(x)=x, \quad L_{k}(x)=\left(2-\frac{1}{k}\right) x L_{k-1}(x)-\left(1-\frac{1}{k}\right) L_{k-2}(x) .
$$

Согласно [1, часть 2 , отдел 6 , задача 93, с. 104, 311]

$$
\begin{gathered}
L_{2 n+1}(\cos x)=c_{0} \cos x+c_{1} \cos 3 x+\cdots+c_{n} \cos (2 n+1) x, \\
c_{0}=2 g_{n} g_{n+1}, c_{1}=2 g_{n-1} g_{n+2}, \ldots, c_{n-1}=2 g_{1} g_{2 n}, c_{n}=2 g_{0} g_{2 n+1}, \\
g_{0}=1, \quad g_{k}=\frac{1 \cdot 3 \cdots(2 k-1)}{2 \cdot 4 \cdots 2 k}(k=1,2,3, \ldots) .
\end{gathered}
$$

Ввиду (7) коэффициенты в (6) положительны и возрастают, т.е. $L_{2 n+1}(\cos x) \in K_{1, n}$; аналогично устанавливается, что $L_{2 n}(\cos x) \in K_{0, n}$. Отсюда следует, что все расстояния между соседними корнями $L_{n}(\cos x)$ имеют один и тот же порядок $O(1 / n)$.

\section{СПИСОК ЛИТЕРАТУРЫ}

[1] Полиа Г., Сеге Г. Задачи и теоремы из анализа. М.: Наука, 1978.

Международньй институт теории прогноза землетрясений и математической геофизики $\mathrm{PAH}$

Принято редколлегией 27.12 .1995 